Pacific Journal of Mathematics

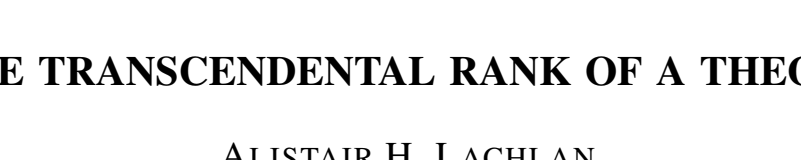




\title{
THE TRANSCENDENTAL RANK OF A THEORY
}

\author{
ALISTAIR H. LACHLAN
}

\begin{abstract}
Morley has associated with each countable complete theory $T$ an ordinal $\alpha_{T}<\left(2^{\boldsymbol{N}_{0}}\right)^{+}$. It is shown that in fact $\alpha_{T} \leqq \omega_{1}$ and that this bound is best possible.
\end{abstract}

We shall use the notation and terminology of Morley [1], where $\alpha_{T}$ is defined to be the least ordinal $\alpha$ such that for all $A \in N(T)$ and all $\beta>\alpha, S^{\alpha}(A)=S^{\beta}(A)$. As in [1] $T$ denotes a complete theory in a countable language $L, T$ has an infinite model, and there is a theory $\Sigma$ such that $T=\Sigma^{*}$. If $A \in N(T)$ and $p \in S(A)$, let $r(p)=\alpha$ if $p$ is transcendental in rank $\alpha$ and let $r(p)$ be undefined otherwise. Also, if $A \in N(T)$ and $\psi \in F(A)$ define

$$
r(\psi, A)=\left\{\begin{array}{l}
-1 \text { if } U_{\psi}=\varnothing \\
\sup \left\{\alpha \mid p \in U_{\psi} \& r(p)=\alpha\right\} \quad \text { otherwise. }
\end{array}\right.
$$

Lemma. Let $A \in N(T), \psi \in F(A)$, and $r(\psi, A)=\alpha$. Then for each $\beta<\alpha$ there exists $B \in N(T), A \subseteq B$, and $q \in S(B)$ such that $r(q)=\beta$ and $\psi \in q$.

Proof. Assume the hypothesis and for contradiction that no $B$ and $q$ exist satisfying the conclusion. Then for every $B \in N(T), A \subseteq B$, we have $i_{A B}^{*-1}\left(U_{\psi}\right) \cap \operatorname{Tr}^{\beta}(B)=\varnothing$. Thus for all such $B, i_{A B}^{*-1}\left(U_{\psi}\right) \cap\left(S^{\beta+1}\right)(B)-$ $\left.S^{\beta}(B)\right)=\varnothing$. Suppose $q^{\prime} \in \operatorname{Tr}^{\beta+1}(B)$ then for every $C \in N(T), B \cong$ $C, i_{B C}^{*-1}\left(q^{\prime}\right) \cap S^{\beta+1}(C)$ is a set of isolated points in $S^{\beta+1}(C)$. Thus, if $\psi \in q^{\prime}, i_{B C}^{*-1}\left(q^{\prime}\right) \cap S^{\beta}(C)$ is a set of isolated points in $S^{\beta}(C)$ for all such $C$, whence $q^{\prime} \in \operatorname{Tr}^{\beta}(B)$. We conclude that $i_{A B}^{*-1}\left(U_{\psi}\right) \cap \operatorname{Tr}^{\beta+1}(B)=\varnothing$ for all $B \in N(T), A \subseteq B$. By induction $i_{A B}^{*-1}\left(U_{\psi}\right) \cap \operatorname{Tr}^{r}(B)=\varnothing$ for all $\gamma \geqq \beta$. This contradicts the hypothesis and completes the proof of the lemma.

From 2.3(b) and 2.4 of [1] it is possible to choose $B$ in the conclusion of the lemma such that $\kappa(B-A)=\aleph_{0}$; we shall make use of this fact below.

Before proceeding further we need some more definitions. A language $L_{1}$ is said to be a simple extension of a language $L_{0}$ if it is obtained by adjoining $\boldsymbol{\aleph}_{0}$ individual constants to $L_{0}$. For any language $L^{\prime}$ let $F\left(L^{\prime}\right)$ denote the set of formulas of $L^{\prime}$ which have no free variable other than $v_{0}$. For each $n \in \omega$ let $S_{n}$ denote the set of all sequences of 0 's and 1's of length $\leqq n$; the empty sequence $\varnothing$ is allowed. For $s \in S_{n}$ and $i \leqq 1, s *\langle i\rangle$ denotes the member of $S_{n+1}$ 
obtained by juxtaposing $i$ to the right of $s$. A map $\psi: S_{n} \rightarrow F(L)$ is called admissible if either $n=0$, or $n>0$ and for each $s \in S_{m}, 0 \leqq m<n$ there exists $\varphi \in F(L)$ such that $\psi(s *\langle 0\rangle)=\psi(s) \& \varphi$ and $\psi(s *\langle 1\rangle)=$ $\psi(s) \& \neg \varphi$. The main step in our proof is:

Proposition. Let $A \in N(T), \kappa(A) \leqq \boldsymbol{\aleph}_{0}$, and $n \in \omega$. Let $\psi_{n}: S_{n} \rightarrow$ $F\left(L_{n}\right)$ be an admissible map, where $L_{n}$ is a simple extension of $L(A)$, such that for every $\alpha<\omega_{1}$ there exists $B_{n}^{\alpha} \in N(T)$ with $A \subseteq B_{n}^{\alpha}$ and $L\left(B_{n}^{\alpha}\right)=L_{n}$ such that for all $s \in S_{n} r\left(\psi_{n}(s), B_{n}^{\alpha}\right) \geqq \alpha$. Then there exists a language $L_{n+1}$, which is a simp'e extension of $L_{n}$ and an admissible map $\psi_{n+1}: S_{n+1} \rightarrow F\left(L_{n+1}\right)$ extending $\psi_{n}$ such that for every $\alpha<\omega_{1}$ there exists $B_{n+1}^{\alpha} \in N(T)$ with $A \subseteq B_{n+1}^{\alpha}$ and $L\left(B_{n+1}^{\alpha}\right)=L_{n+1}$ such that for all $s \in S_{n+1}, r\left(\psi_{n+1}(s), B_{n+1}^{\alpha}\right) \geqq \alpha$.

Proof. Form $L_{n+1}$ by adjoining a countable number of new individual constants to $L_{n}$. Consider a fixed ordinal $\alpha<\omega_{1}$. By $2^{n+1}$ applications of the lemma we can find $C^{\alpha} \in N(T)$ with $B_{n}^{\alpha+2} \subseteq C^{\alpha}$ and $L\left(C^{\alpha}\right)=L_{n+1}$ such that for each $s \in S_{n}-S_{n-1}$ there exist $p_{0}(s), p_{1}(s) \in$ $S\left(C^{\alpha}\right)$ both containing $\psi_{n}(s)$ such that $r\left(p_{0}(s)\right)=\alpha$ and $r\left(p_{1}(s)\right)=\alpha+1$. For each $s \in S_{n}-S_{n-1}$ choose $\varphi^{\alpha}(s) \in p_{0}(s)-p_{1}(s)$. Define $\psi^{\alpha}: S_{n+1} \rightarrow$ $F\left(L_{n+1}\right)$ to be the extension of $\psi_{n}$ such that for each $s \in S_{n}-S_{n-1}$, $\psi^{\alpha}(s *\langle 0\rangle)=\psi_{n}(s) \& \varphi^{\alpha}(s)$ and $\psi^{\alpha}(s *\langle 0\rangle)=\psi_{n}(s) \& \neg \varphi^{\alpha}(s)$. Letting $\psi_{n+1}=$ $\psi^{\alpha}$ and $B_{n+1}^{\alpha}=C^{\alpha}$ the conclusion of the lemma holds for $\alpha$. Perform the construction of $\psi^{\alpha}$ for each $\alpha<\omega_{1}$. Since $L_{n+1}$ is countable the set $\left\{\psi^{\alpha} \mid \alpha<\omega_{1}\right\}$ is countable. Hence there is a cofinal subset $\Gamma$ of $\omega_{1}$ such that $\psi^{r}$ is independent of $\gamma$ for $\gamma \in \Gamma$. Let $\psi_{n+1}$ be the common value of $\psi^{\gamma}$ for $\gamma \in \Gamma$. For each $\alpha<\omega_{1}$ let $\gamma$ be the least member of $\Gamma$ such that $\alpha<\gamma$ and define $B_{n+1}^{\alpha}=C^{\gamma}$. This completes the proof of the proposition.

Let $S_{\omega}$ denote the set of all finite sequences of 0 's and 1's. A sequence $\left\langle s_{i}\right\rangle_{i<\omega}$ of members of $S_{\omega}$ is called regular if $s_{0}=\varnothing$ and for all $i\left\langle\omega, s_{i+1}\right.$ is either $s_{i} *\langle 0\rangle$ or $s_{i} *\langle 1\rangle$. Now let $A \in N(T)$ with $\kappa(A) \leqq \boldsymbol{\aleph}_{0}$, and let $p \in S(A)$ with $r(p)=\omega_{1}$. Choose $\varphi \in F(A)$ such that $U_{\varphi} \cap S^{\omega_{1}}(A)=\{p\}$. Let $L_{0}$ be $L(A)$ and define $\psi_{0}: S_{0} \rightarrow F\left(L_{0}\right)$ by $\psi_{0}(\varnothing)=\varnothing$ then $\varphi_{0}$ is admissible. Apply the proposition repeatedly to form $L_{1}, L_{2}, \cdots$ and $\psi_{1}, \psi_{2}, \cdots$. Let $L_{\omega}=U_{n<\omega} L_{n}$ and let $\psi=\lim _{n<\omega} \psi_{n}$ where $\psi$ maps $S_{\omega}$ into $F\left(L_{\omega}\right)$. By the compactness theorem there exists $B \in N(T)$ such that $A \subseteq B, \kappa(B)=\boldsymbol{\aleph}_{0}, L(B)=L_{\omega}$, and such that if $\left\langle s_{i}\right\rangle_{i<\omega}$ is a regular sequence in $S_{\omega}$ then $\left\{\psi\left(s_{i}\right) \mid i<\omega\right\} \subseteq q$ for some $q \in S(B)$. Let $s \in S_{\omega}$ then it is clear that the basic open set $U_{\psi(s)}$ of $S(B)$ has power $2^{\aleph_{0}}$. Also, since $\kappa(B)=\aleph_{0}$, for every $\alpha S^{\alpha+1}(B)-S^{\alpha}(B)$ is countable. Thus $U_{\psi(s)} \cap S^{\alpha}(B) \neq \varnothing$ for all $\alpha<\omega_{1}$. Since $S^{\alpha}(B)$ is closed and decreasing with $\alpha, U_{\psi(s)} \cap S^{\omega_{1}}(B) \neq \varnothing$. It follows immedia- 
tely that $\kappa\left(U_{\varphi}^{\prime} \cap S^{\omega_{1}}(B)\right) \geqq \boldsymbol{\aleph}_{0}$ where $U_{\varphi}^{\prime}$ denotes the basic open set of $S(B)$ determined by $\varphi$. From 2.3(b) of [1] $i_{A B}^{*}\left(S^{\omega_{1}}(B)\right)=S^{\omega_{1}}(A)$. Since $i_{A B}^{*}\left(U_{\varphi}^{\prime}\right)=U_{\varphi}$ it follows that $i_{A B}^{*-1}(p)=U_{\varphi}^{\prime} \cap S^{\omega_{1}}(B)$. But this contradicts $r(p)=\omega_{1}$ because $U_{\varphi}^{\prime} \cap S^{\omega_{1}}(B)$ having power $\geqq \aleph_{0}$ is not a set of isolated points.

Since $\operatorname{Tr}^{\alpha}(A) \neq \varnothing$ for some finite $A \in N(T)$ if for any $A \in N(T)$, we have shown that $\operatorname{Tr}^{\omega_{1}}(A)=\varnothing$ for every $A \in N(T)$. It follows easily that $S^{\beta}(A)=S^{\omega_{1}}(A)$ for every $\beta>\omega_{1}$ and every $A \in N(T)$. Thus $\alpha_{T} \leqq \omega_{1}$ and our main theorem is proved.

We shall now construct a theory $T$ such that $\alpha_{T}=\omega_{1} \cdot{ }^{1}$ In Example III of $\S 2$ of [1] Morley showed how to construct a theory $T_{\beta}$ for any $\beta<\omega_{1}$ such that $\alpha_{T_{\beta}}=\beta+1$ and such that $L\left(T_{\beta}\right)=$ $\left\{R_{n} \mid n<\omega\right\}$ where each $R_{n}$ is a unary relation symbol. For $\beta<\omega_{1}$ let $A_{\beta}$ be a model of $T_{\beta}$. Suppose without loss that the sets $\left|A_{\beta}\right|$, $\beta<\omega_{1}$, are pairwise disjoint and each disjoint from $\omega_{1}$. Now let $A$ be the relational system such that $|A|=\omega_{1} \cup_{\beta<\omega_{1}}\left|A_{\beta}\right|$ and define relations $R^{A}, R_{0}^{A}, R_{1}^{A}, \cdots$ as follows: for all $x, y \in|A|$

and

$$
R^{A}(x, y) \Longleftrightarrow x \in \omega_{1} \& y \in\left|A_{x}\right|
$$

$$
R_{n}^{A}(y) \Longleftrightarrow \bigvee x\left(x \in \omega_{1} \& y \in R_{n}^{A x}\right)
$$

If $T$ is the theory of the system $A$ then it is easy to see that $\alpha_{T}=\omega_{1}$. In fact $\alpha_{T}$ can have as its value any ordinal $\leqq \omega_{1}$ other than 0 . From the examples to be found above it is sufficient to treat the case in which $\beta$ is a limit ordinal $\left\langle\omega_{1}\right.$. Let $\left\langle\beta_{n}\right\rangle_{n<\omega}$ be a strictly increasing sequence with limit $\beta$. Let $T^{\#}$ be the theory with the same language as $T_{\beta}$ above such that if $A$ is any model of $T^{*}$ and $F, G$ are disjoint finite subsets of $\omega$ then

$$
\bigcap\left\{R_{n}^{A} \mid n \in F\right\} \cap \bigcap\left\{|A|-R_{n}^{A} \mid n \in G\right\} \neq \varnothing .
$$

Choose axioms $\psi_{0}, \psi_{1}, \cdots$ for $T^{\sharp}$ which are all existential, this is easy to do. For each $n$ modify the theory $T_{\beta_{n}}$ to obtain a theory $T_{n}^{\prime}$ whose transcendental rank is $\beta_{n}+1$ and which has $\psi_{0}, \psi_{1}, \cdots, \psi_{n-1}$ amongst its theorems. For each $n<\omega$ let $A_{n}$ be a model of $T_{n}$. Suppose that the sets $\left|A_{n}\right|, n<\omega$, are pairwise disjoint and disjoint from $\omega$. Now let $A$ be the relational system such that $|A|=\omega \cup \bigcup_{n<\omega}\left|A_{n}\right|$ with relations $R^{A}, R_{0}^{A}, R_{1}^{A}, \cdots$ defined by

and

$$
R^{A}(x, y) \Longleftrightarrow x \in \omega \& y \in\left|A_{x}\right|
$$

$$
R_{n}^{A}(y) \Longleftrightarrow \mathrm{V} x\left(x \in \omega \& y \in R_{n}^{A} x\right) \text {. }
$$

1 The referee informs me that similar examples have been found independently by several people. 
If $T$ is the theory of the system $A$ then it is easy to see that $\alpha_{T}=\beta$.

\section{REFERENCES}

1. M. Morley, Categoricity in power, Trans. Amer. Math. Soc., 114 (1965), 514-538. Received April 23, 1970.

Simon Fraser University 


\section{PACIFIC JOURNAL OF MATHEMATICS}

\section{EDITORS}

H. SAMELSON

Stanford University

Stanford, California 94305

C. R. Новву

University of Washington

Seattle, Washington 98105
J. DUGundJI

Department of Mathematics

University of Southern California

Los Angeles, California 90007

RICHARD ARENS

University of California

Los Angeles, California 90024

\section{ASSOCIATE EDITORS}
E. F. BECKENBACH
B. H. NeumanN
F. WOLF
K. YOSHIDA

\section{SUPPORTING INSTITUTIONS}
UNIVERSITY OF BRITISH COLUMBIA
CALIFORNIA INSTITUTE OF TECHNOLOGY
UNIVERSITY OF CALIFORNIA
MONTANA STATE UNIVERSITY
UNIVERSITY OF NEVADA
NEW MEXICO STATE UNIVERSITY
OREGON STATE UNIVERSITY
UNIVERSITY OF OREGON
OSAKA UNIVERSITY
UNIVERSITY OF SOUTHERN CALIFORNIA
STANFORD UNIVERSITY
UNIVERSITY OF TOKYO
UNIVERSITY OF UTAH
WASHINGTON STATE UNIVERSITY
UNIVERSITY OF WASHINGTON
AMERICAN MATHEMATICAL SOCIETY CHEVRON RESEARCH CORPORATION TRW SYSTEMS
NAVAL WEAPONS CENTER 


\section{Pacific Journal of Mathematics}

\section{Vol. 37, No. $1 \quad$ January, 1971}

Gregory Frank Bachelis and Haskell Paul Rosenthal, On unconditionally

converging series and biorthogonal systems in a Banach space .........

Richard William Beals, On spectral theory and scattering for elliptic

operators with singular potentials .........................

J. Lennart (John) Berggren, Solvable and supersolvable groups in which every element is conjugate to its inverse ........................ 21

Lindsay Nathan Childs, On covering spaces and Galois extensions ..........

William Jay Davis, David William Dean and Ivan Singer, Multipliers and

unconditional convergence of biorthogonal expansions..............

Leroy John Derr, Triangular matrices with the isoclinal property ............

Paul Erdős, Robert James McEliece and Herbert Taylor, Ramsey bounds for

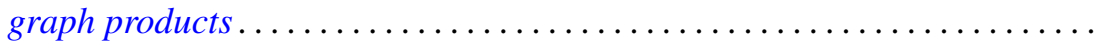

Edward Graham Evans, Jr., On epimorphisms to finitely generated

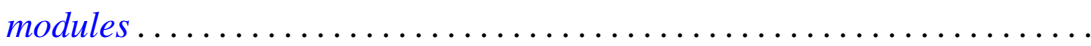

Hector O. Fattorini, The abstract Goursat problem ................. 51

Robert Dutton Fray and David Paul Roselle, Weighted lattice paths .........

Thomas L. Goulding and Augusto H. Ortiz, Structure of semiprime $(p, q)$

radicals ...........................................

E. W. Johnson and J. P. Lediaev, Structure of Noether lattices with join-principal maximal elements ....

David Samuel Kinderlehrer, The regularity of minimal surfaces defined over

slit domains

Alistair H. Lachlan, The transcendental rank of a theory. .

Frank David Lesley, Differentiability of minimal surfaces at the boundary ...

Wolfgang Liebert, Characterization of the endomorphism rings of divisible torsion modules and reduced complete torsion-free modules over complete discrete valuation rings....

Lawrence Carlton Moore, Strictly increasing Riesz norms.

Raymond Moos Redheffer, An inequality for the Hilbert transform ...

James Ted Rogers Jr., Mapping solenoids onto strongly self-entwined,

circle-like continua..........................

Sherman K. Stein, B-sets and planar maps ................... 217

Darrell R. Turnidge, Torsion theories and rings of quotients of Morita

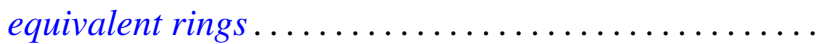

Fred Ustina, The Hausdorff means of double Fourier series and the principle of localization ................................

Stanley Joseph Wertheimer, Quasi-compactness and decompositions for arbitrary relations.

Howard Henry Wicke and John Mays Worrell Jr., On the open continuous images of paracompact $\check{C}$ ech complete spaces... 